\title{
A NOTE ON ASYMPTOTICALLY GOOD EXTENSIONS IN WHICH INFINITELY MANY PRIMES SPLIT COMPLETELY
}

\author{
by \\ Oussama Hamza \& Christian Maire
}

\begin{abstract}
Let $p$ be a prime number, and let $\mathrm{K}$ be a number field. For $p=2$, assume moreover $\mathrm{K}$ totally imaginary. In this note we prove the existence of asymptotically good extensions L/K of cohomological dimension 2 in which infinitely many primes split completely. Our result is inspired by a recent work of Hajir, Maire, and Ramakrishna [7].
\end{abstract}

Let $\mathrm{K}$ be a number field, and let $\mathrm{L} / \mathrm{K}$ be an infinite unramified extension. Denote by $\mathscr{S}_{\mathrm{L} / \mathrm{K}}$ the set of prime ideals of $\mathrm{K}$ that split completely in $\mathrm{L} / \mathrm{K}$. In [8] Ihara proved that $\sum_{\mathfrak{p} \in \mathscr{S}_{\mathrm{L} / \mathrm{K}}} \frac{\log \mathrm{N}(\mathfrak{p})}{\mathrm{N}(\mathfrak{p})}<\infty$, and raised the following interesting question: are there $\mathrm{L} / \mathrm{K}$ for which $\mathscr{S}_{\mathrm{L} / \mathrm{K}}$ is infinite ? This question was recently answered in the positive by Hajir, Maire, and Ramakrishna in [7]. In fact, infinite unramified extensions $\mathrm{L} / \mathrm{K}$ are some special cases of infinite extensions for which the root discriminants $\operatorname{rd}_{\mathrm{F}}:=\left|\operatorname{Disc}_{\mathrm{F}}\right|^{1 /[\mathrm{F}: \mathbb{Q}]}$ are bounded, where the number fields $\mathrm{F}$ vary in $\mathrm{L} / \mathrm{K}$, and $\mathrm{Disc}_{\mathrm{F}}$ is the discriminant of $\mathrm{F}$. Such extensions are called asymptotically good, and it is now well-known that in such extensions the inequality of Ihara involving $\mathscr{S}_{\mathrm{L} / \mathrm{K}}$ still holds (see for example [16], or [13] for the study of such extensions).

Pro- $p$ extensions of number fields with restricted ramification allow us to exhibit asymptotically good extensions. Let $p$ be a prime number, and let $S$ be a finite set of prime ideals of $\mathrm{K}$ coprime to $p$ (more precisely each $\mathfrak{p} \in S$ is such that $\left|\mathscr{O}_{\mathrm{K}} / \mathfrak{p}\right| \equiv 1(\bmod p)$ ); the set $S$ is called tame. Let $\mathrm{K}_{S}$ the maximal pro- $p$ extension of $\mathrm{K}$ unramified outside $S$, put $\mathrm{G}_{S}=\mathrm{Gal}\left(\mathrm{K}_{S} / \mathrm{K}\right)$. In $\mathrm{K}_{S} / \mathrm{K}$ the root discriminants are bounded by some constant depending on the discriminant of $\mathrm{K}$ and the norm of the places of $S$ (see for example [6. Lemma 5]). Moreover thanks to Golod-Shafarevich criterion, it is well-known that $\mathrm{K}_{S} / \mathrm{K}$ is infinite when $|S|$ is large as compared to $[\mathrm{K}: \mathbb{Q}]$ (see for example [14, Chapter $\mathrm{X}, \S 10$, Theorem 10.10.1]), and then asymptotically good. E.g. for $p>2, \mathbb{Q}_{S} / \mathbb{Q}$ is infinite when $|S| \geqslant 4$. In [7] the authors showed that when $S$ is large, there exist infinite subextension

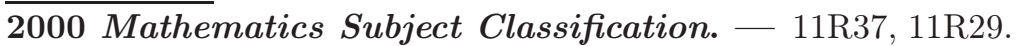

Key words and phrases. - Pro- $p$ extensions with restricted ramification, asymptotically good extensions, mild pro- $p$ extensions.

The authors thank Farshid Hajir for useful comments, and Philippe Lebacque for his interest in this work. CM was partially supported by the ANR project FLAIR (ANR-17-CE40-0012), and by the EIPHI Graduate School (ANR-17-EURE-0002). 
$\mathrm{L} / \mathrm{K}$ of $\mathrm{K}_{S} / \mathrm{K}$ for which the set $\mathscr{S}_{\mathrm{L} / \mathrm{K}}$ is infinite. But they give no information about the structure of $\mathrm{Gal}(\mathrm{L} / \mathrm{K})$. Here we prove:

Theorem A. - Let $p$ be a prime number, and let $\mathrm{K}$ be a number field. For $p=2$ assume $\mathrm{K}$ totally imaginary. Let $T$ and $S_{0}$ be two disjoint finite sets of prime ideals of $\mathrm{K}$ where $S_{0}$ is tame. Then for infinitely many finite sets $S$ of tame prime ideals of $\mathrm{K}$ containing $S_{0}$ there exist an infinite pro-p extension $\mathrm{L} / \mathrm{K}$ in $\mathrm{K}_{S} / \mathrm{K}$ such that

(i) the set $\mathscr{S}_{\mathrm{L} / \mathrm{K}}$ of places that split completely in $\mathrm{L} / \mathrm{K}$ contains $T$;

(ii) the set $\mathscr{S}_{\mathrm{L} / \mathrm{K}}$ is infinite;

(iii) the pro-p group $\mathrm{G}=\mathrm{Gal}(\mathrm{L} / \mathrm{K})$ is of cohomological dimension 2 ;

(iv) the minimal number of relations of $\mathrm{G}$ is infinite, i.e. $\operatorname{dim} H^{2}\left(\mathrm{G}, \mathbb{F}_{p}\right)=\infty$;

(v) for each $\mathfrak{p} \in S$, the local extension $\mathrm{L}_{\mathfrak{p}} / \mathrm{K}_{\mathfrak{p}}$ is maximal, i.e. isomorphic to $\mathbb{Z}_{p} \rtimes \mathbb{Z}_{p}$;

(vi) the Poincaré series of the algebra $\mathbb{F}_{p} \llbracket \mathrm{G} \rrbracket$, endowed with the graduation from the ideal of augmentation, is equal to $\left(1-d t+r t^{2}+t^{3} \sum_{n \geqslant 0} t^{n}\right)^{-1}$, where $d=\operatorname{dim} \mathrm{G}_{S}$, and where $r$ is explicit, depending on $\mathrm{K}, S, T$.

Remark 1. - We will see that the pro-p group $\mathrm{G}$ of Theorem $A$ is mild in the terminology of Anick [2]. See also Labute [10] for arithmetic contexts.

The proof uses various tools.

The first one is the strategy developed initially by Labute [10], then by Labute-Mináč [1], Schmidt [15], Forré [4] etc. for studying the cohomological dimension of a pro- $p$ group $\mathrm{G}$, through the notion of strongly free sets introduced by Anick [1]. By following the approach of Forré [4, we refine this idea when the minimal number of relations of $\mathrm{G}$ is infinite.

This key idea is associated to a result of Schmidt [15] that shows that the pro- $p$ group $\mathrm{G}_{S}$ is of cohomological dimension 2 for some well-chosen $S$; the proof of Schmidt involves the cup-product $H^{1}\left(\mathrm{G}_{S}, \mathbb{F}_{p}\right) \cup H^{1}\left(\mathrm{G}_{S}, \mathbb{F}_{p}\right)$. Here we use the translation of this cup-product in the polynomial algebra, due to Forré. In particular, this allows us to choose infinitely many Frobenius in $\mathrm{G}_{S}$ such that the family of the highest terms of these plus the highest terms of the relations of $\mathrm{G}_{S}$, is combinatorially free (see 1.1 .3 and Definition 1.2).

We conclude by cutting the tower $\mathrm{K}_{S} / \mathrm{K}$ by all these Frobenius: this is the strategy of [7].

This note contains two sections. In $\S 1$ we recall the results we need regarding pro- $p$ groups, graded algebras, and arithmetic of pro- $p$ extensions with restricted ramification. In $\S 2$ we start with an example when $K=\mathbb{Q}$, and prove the main result.

\section{Notations.}

Let $p$ be a prime number.

- If $\mathrm{V}$ is a $\mathbb{F}_{p^{-}}$-vector space we denote by $\operatorname{dim} \mathrm{V}$ its dimension over $\mathbb{F}_{p}$.

- For a pro- $p$ group $\mathrm{G}$, we denote by $H^{i}(\mathrm{G})$ the cohomology group $H^{i}\left(\mathrm{G}, \mathbb{F}_{p}\right)$. The $p$-rank of $\mathrm{G}$, which is equal to $\operatorname{dim} H^{1}(\mathrm{G})$, is noted $d_{p} \mathrm{G}$.

\section{The results we need}

1.1. On pro- $p$ groups. - For this section we refer to [3], [9, Chapters 5,6 and 7], and 4. Take a prime number $p$. 
1.1.1. Minimal presentation and cohomological dimension. - Let $\mathrm{G}$ be a pro- $p$ group of finite rank $d$, and let $1 \rightarrow \mathrm{R} \rightarrow \mathrm{F} \rightarrow \mathrm{G} \rightarrow 1$ be a minimal presentation of $\mathrm{G}$ by a free pro- $p$ group $\mathrm{F}$. Let $\mathscr{F}:=\left\{\rho_{i}\right\}_{i \in I}$ be an $\mathbb{F}_{p}$-basis of $\mathrm{R} / \mathrm{R}^{p}[\mathrm{~F}, \mathrm{R}]$; observe that $I$ is not necessarily finite. The algebra $\Lambda_{\mathrm{G}}:=\mathbb{F}_{p} \llbracket \mathrm{G} \rrbracket$ acts on $\mathrm{R} / \mathrm{R}^{p}[\mathrm{R}, \mathrm{R}]$, and by Nakayama's lemma the $\rho_{i}$ 's generate topologically $\mathrm{R} / \mathrm{R}^{p}[\mathrm{R}, \mathrm{R}]$ as $\Lambda_{\mathrm{G}}$-module (see for example [3, Corollary 1.5]).

Let us recall the definition of the cohomological dimension $\mathrm{cd}(\mathrm{G})$ of $\mathrm{G}$ : it is the smallest integer $n$ (eventually $n=\infty$ ) such that $H^{i}(\mathrm{G})=0$ for every $i \geqslant n+1$.

Theorem 1.1. - The following assertions are equivalent:

(i) $\operatorname{cd}(\mathrm{G}) \leqslant 2$;

(ii) $\mathrm{R} / \mathrm{R}^{p}[\mathrm{R}, \mathrm{R}]$ is a free compact $\Lambda_{\mathrm{G}}$-module;

(iii) $\mathrm{R} / \mathrm{R}^{p}[\mathrm{R}, \mathrm{R}] \simeq \prod_{I} \Lambda_{\mathrm{G}}$.

Moreover, $\operatorname{dim} H^{2}(\mathrm{G})=|I|$.

Proof. - See [3, Corollary 5.3] or [9, Chapter 7, §7.3, Theorem 7.7].

We are going to translate conditions of Theorem 1.1 in the algebra $\mathbb{F}_{p}^{n c} \llbracket X_{1}, \cdots, X_{d} \rrbracket$.

1.1.2. Filtred and graded algebras. - The results of this section can be found in [1].

- Let

$$
\mathrm{E}=\mathbb{F}_{p}^{n c} \llbracket X_{1}, \cdots, X_{d} \rrbracket
$$

be the algebra of noncommutative series in $X_{1}, \cdots, X_{d}$ with coefficients in $\mathbb{F}_{p}$. We consider now noncommutative multi-indices $\alpha=\left(\alpha_{1}, \cdots, \alpha_{n}\right)$, with $\alpha_{i} \in\{1, \cdots, d\}$, and we denote by $X_{\alpha}$ the monomial element of the form $X_{\alpha}=X_{\alpha_{1}} \cdots X_{\alpha_{n}}$. We endow each $X_{i}$ with the degree 1 ; the degree $\operatorname{deg}\left(X_{\alpha}\right)$ of $X_{\alpha}$ is $|\alpha|$.

For $Z=\sum_{\alpha} a_{\alpha} X_{\alpha}$, the quantity $\omega(Z)=\min _{a_{\alpha} \neq 0}\left\{\operatorname{deg}\left(X_{\alpha}\right)\right\}$ is the valuation of $Z$, with the convention that $\omega(0)=\infty$. For $n \geqslant 0$, put $\mathrm{E}_{n}=\{Z \in \mathrm{E}, \omega(Z) \geqslant n\}$. Observe that $\mathrm{E}_{1}$ is the augmentation ideal of $\mathrm{E}$ : this is the two-sided ideal of $\mathrm{E}$ topologically generated by the $X_{i}$ 's. The algebra $\mathrm{E}$ is filtered by the $\mathrm{E}_{n}$ 's and its graded algebra $\operatorname{Grad}(\mathrm{E})$ is then:

$$
\operatorname{Grad}(\mathrm{E})=\bigoplus_{n \in \mathbb{Z} \geqslant 0} \mathrm{E}_{n} / \mathrm{E}_{n+1} \simeq \mathbb{F}_{p}^{n c}\left[X_{1}, \ldots, X_{d}\right]
$$

In other words $\operatorname{Grad}(\mathrm{E})$ is isomorphic to the noncommutative polynomial algebra $\mathrm{A}:=$ $\mathbb{F}_{p}^{n c}\left[X_{1}, \ldots, X_{d}\right]$, where each $X_{i}$ is endowed with the formal degree 1 . Let $\mathrm{A}_{n}=\{z \in$ $\mathrm{A}, \omega(z) \geqslant n\}$ be the gradation of $\mathrm{A}$; observe that $\mathrm{A}_{1}$ is the augmentation ideal of $\mathrm{A}$.

- Let $X_{\alpha}, X_{\alpha^{\prime}}$ be two monomials (viewed in E or in A). The element $X_{\alpha}$ is a submonomial of $X_{\alpha^{\prime}}$, if $X_{\alpha^{\prime}}=X_{\beta} X_{\alpha} X_{\beta^{\prime}}$, with $X_{\beta}, X_{\beta^{\prime}}$ two monomials of A.

Definition 1.2. - A family $\mathscr{F}=\left\{X_{\alpha^{(i)}}\right\}_{i \in I}$ of monomials of A is combinatorially free if for all $i \neq j$ :

(i) $X_{\alpha^{(i)}}$ is not a submonomial of $X_{\alpha^{(j)}}$,

(ii) if $X_{\alpha^{(i)}}=X_{\alpha} X_{\beta}$ and $X_{\alpha^{(j)}}=X_{\alpha^{\prime}} X_{\beta^{\prime}}$, then $X_{\alpha} \neq X_{\alpha^{\prime}}$, with $X_{\alpha}, X_{\beta}, X_{\alpha^{\prime}}, X_{\beta^{\prime}}$ non-trivial monomials, i.e. $\neq 1$.

The monomials may be endowed with a total order $<$ as follows.

First let us consider the natural ordering $<^{\prime}$ defined by: $X_{1}<^{\prime} X_{2}<^{\prime} \cdots<^{\prime} X_{d}$. 
Let $X_{\alpha}$ and $X_{\beta}$ two monomials, we say that $X_{\alpha}>X_{\beta}$, if $\omega\left(X_{\alpha}\right)<\omega\left(X_{\beta}\right)$; if $X_{\alpha}$ and $X_{\beta}$ have the same valuation, we use the lexicographic order induced by $<^{\prime}$.

Now, let $Z=\sum_{\alpha} a_{\alpha} X_{\alpha}$ be a nonzero element of E, with $a_{\alpha} \in \mathbb{F}_{p}$. Then $\hat{Z}:=$ $\max \left\{X_{\alpha}, a_{\alpha} \neq 0\right\}$ is the highest term respecting the order $<$.

- Let $\mathscr{F}:=\left\{Z_{i}\right\}_{i \in I}$ be a locally finite graded subset of $\mathrm{A}_{1}$ generating $\mathrm{C}$ as two-sided A-ideal: $\mathrm{C}=\mathrm{A} \mathscr{F} \mathrm{A}$. Observe that $I$ is countable. Let $\mathrm{B}:=\mathrm{A} / \mathrm{C}$ be the quotient endowed with the quotient gradation; we denote by $P_{\mathrm{B}}(t)=\sum_{n \in \mathbb{Z} \geqslant 0} \operatorname{dim}\left(\mathrm{B}_{n} / \mathrm{B}_{n+1}\right) \cdot t^{n}$ the Poincaré series of $\mathrm{B}$. Observe that the family $\mathscr{F}$ generates the $\mathrm{B}$-module $\mathrm{C} / \mathrm{CA}_{1}$.

Theorem 1.3 (Anick). - Let $\mathscr{F}=\left\{Z_{i}\right\}_{i \in I}$ be a locally finite graded subset of $\mathrm{A}_{1}$, and let $\mathrm{C}$ be a two-sided ideal of $\mathrm{A}$ generated by the $Z_{i}$ 's; put $\mathrm{B}=\mathrm{A} / \mathrm{C}$. For each $i$, let $X_{\alpha^{(i)}}:=\widehat{Z}_{i}$ be the highest term of $Z_{i}$. If the family $\left\{X_{\alpha^{(i)}}\right\}_{i \in I}$ is combinatorially free, then

(i) $\mathrm{C} / \mathrm{CA}_{1}$ is a free $\mathrm{B}$-module over the $Z_{i}$ 's, and

(ii) $P_{\mathrm{B}}(t)=\left(1-d t+\sum_{i \in I} t^{n_{i}}\right)^{-1}$, where $n_{i}=\omega\left(Z_{i}\right)=\omega\left(X_{\alpha^{(i)}}\right)$.

Proof. - See [1, Theorems 2.6 and 3.2].

If $\mathrm{C} / \mathrm{CA}_{1}$ is a free $\mathrm{B}$-module over the $Z_{i}$ 's, we say that the family $\mathscr{F}=\left\{Z_{i}\right\}_{i \in I}$ is strongly free (see [1]).

Example 1.4. - Take $d=5$, and the lexicographic ordering $X_{1}<X_{2}<\cdots<$ $X_{5}$. Let $a_{n} \geqslant 1$ be an increasing sequence, $n \geqslant 1$, and consider the family $\mathscr{F}=$ $\left\{X_{5} X_{3}, X_{4} X_{2}, X_{4} X_{3}, X_{5} X_{2}, X_{5} X_{1}, X_{5} X_{4}^{a_{n}} X_{1}, n \geqslant 1\right\}$. Put $\mathrm{B}=\mathrm{A} / \mathrm{A} \mathscr{F} \mathrm{A}$. Then $\mathscr{F}$ is combinatorially free, and $P_{\mathrm{B}}(t)=\left(1-5 t+t^{2} \sum_{n \geqslant 1} t^{a_{n}}\right)^{-1}$.

1.1.3. Pro-p groups of cohomological dimension $\leqslant 2$ and polynomial algebra. - Let us conserve the notations of $\$ 1.1 .1$.

Let $\mathrm{F}$ be a free pro- $p$ group on $d$ generators $x_{1}, \cdots, x_{d}$. Let $\Lambda_{\mathrm{F}}:=\mathbb{F}_{p} \llbracket \mathrm{F} \rrbracket$ be the complete algebra associated to $\mathrm{F}$. Recall that $\mathbb{F}_{p} \llbracket \mathrm{F} \rrbracket$ is isomorphic to the Magnus algebra $\mathrm{E}=$ $\mathbb{F}_{p}^{n c} \llbracket X_{1}, \cdots, X_{d} \rrbracket$; this isomorphism $\varphi$ is given by $x_{i} \mapsto X_{i}+1$ (see for example [9, Chapter 7, §7.6, Theorem 7.16]).

Let us endow $E$ with the filtration and the ordering of $\$ 1.1 .2$. The filtered isomorphism $\varphi$ : $\Lambda_{\mathrm{F}} \stackrel{\simeq}{\rightarrow}$ E allows us to endow $\Lambda_{\mathrm{F}}$ with the valuation $\omega_{\mathrm{F}}$ defined as follows: $\omega_{\mathrm{F}}(z)=\omega(\varphi(z))$. Observe that $\mathrm{E}_{1} \simeq \mathrm{I}_{\mathrm{F}}: \operatorname{ker}\left(\Lambda_{\mathrm{F}} \rightarrow \mathbb{F}_{p}\right)$, that is $\mathrm{E}_{1}$ is isomorphic to the augmentation ideal of $\Lambda_{\mathrm{F}}$.

Take $x \in \mathrm{F}, x \neq 1$. Then the $\operatorname{degree} \operatorname{deg}(x)$ of $x$ is defined as $\operatorname{deg}(x):=\omega_{\mathrm{F}}(x-1)=$ $\omega(\varphi(x-1))$. We denote by $\widehat{x}$ the highest term of $\varphi(x-1) \in$ E. Hence $\widehat{x}$ is a monomial.

Example 1.5. - Take $d \geqslant 3$ with the lexicographic ordering $X_{1}<X_{2}<X_{3}<\cdots<X_{d}$.

(i) The highest term of $\left[x_{1},\left[x_{2}^{p^{n}}, x_{3}\right]\right]$ is $X_{3} X_{2}^{p^{n}} X_{1}$.

(ii) Given $x, y \in \mathrm{F}$, let us write $f_{x}(y)=[x, y] \in \mathrm{F}$. Then the highest term of $f_{x_{1}} \circ$ $f_{x_{2}}^{\circ^{n}}\left(x_{3}\right)$ is $X_{3} X_{2}^{n} X_{1}$.

Let $\mathrm{G}$ be a pro- $p$ group of $p$-rank $d$, and let $1 \rightarrow \mathrm{R} \rightarrow \mathrm{F} \rightarrow \mathrm{G} \rightarrow 1$ be a minimal presentation of $\mathrm{G}$ by $\mathrm{F}$; this induces a filtered morphism $\theta: \Lambda_{\mathrm{F}} \rightarrow \Lambda_{\mathrm{G}}$. We now endow $\Lambda_{\mathrm{G}}$ with the induced valuation $\omega_{\mathrm{G}}$ of $\omega_{\mathrm{F}}$ as follows: for $z \in \Lambda_{\mathrm{G}}$, let us define

$$
\omega_{\mathrm{G}}(z)=\max \left\{\omega_{\mathrm{F}}\left(z^{\prime}\right), z^{\prime} \in \Lambda_{\mathrm{F}}, \theta\left(z^{\prime}\right)=z\right\} .
$$


Put $\mathrm{E}_{\mathrm{G}, n}=\left\{z \in \Lambda_{\mathrm{G}}, \omega_{\mathrm{G}}(z) \geqslant n\right\}$, the filtration of $\Lambda_{\mathrm{G}}$. Then $\operatorname{Grad}\left(\Lambda_{\mathrm{G}}\right)=$ $\oplus_{n} \mathrm{E}_{\mathrm{G}, n} / \mathrm{E}_{\mathrm{G}, n+1}$ is the graded algebra of $\mathbb{F}_{p} \llbracket \mathrm{G} \rrbracket$ respecting the quotient gradation with $P_{\mathrm{G}}(t)=\sum_{n \geqslant 0} \operatorname{dim} \mathrm{E}_{\mathrm{G}, n} / \mathrm{E}_{\mathrm{G}, n+1} \cdot t^{n}$ as Poincaré series.

For $n \geqslant 1$, put $\mathrm{F}_{n}:=\left\{x \in \mathrm{F}, \varphi(x-1) \in \mathrm{E}_{n}\right\}$, and $\mathrm{G}_{n}=\mathrm{F}_{n} \mathrm{R} / \mathrm{R}$. The sequences $\left(\mathrm{F}_{n}\right)$ and $\left(\mathrm{G}_{n}\right)$ are the Zassenhaus filtrations of $\mathrm{F}$ and $\mathrm{G}$. The filtration $\left(\mathrm{E}_{\mathrm{G}, n}\right)$ corresponds also to the filtration coming from the augmentation ideal of $\Lambda_{\mathrm{G}}$ (see for example [12, Appendice A.3, Théorème 3.5]).

Theorem 1.6. - Let $\mathscr{F}=\left\{\rho_{i}\right\}_{i \in I}$ be a family of generators $\mathrm{R} / \mathrm{R}^{p}[\mathrm{R}, \mathrm{R}]$. For each $\in I$, let $X_{\alpha^{(i)}}=\left\{\hat{\rho}_{i}\right\}_{i \in I} \in \mathrm{A}$ be the highest term of $\rho_{i}$. If $\left\{X_{\alpha^{(i)}}\right\}_{i \in I}$ is combinatorially free, then

(i) $\mathrm{R} / \mathrm{R}^{p}[\mathrm{R}, \mathrm{R}] \simeq \prod_{i \in I} \Lambda_{\mathrm{G}}$, and $\operatorname{cd}(\mathrm{G}) \leqslant 2$;

(ii) $P_{\mathrm{G}}(t)=\left(1-d t+\sum_{i \in I} t^{n_{i}}\right)^{-1}$, where $d=d_{p} \mathrm{G}$, and $n_{i}=\operatorname{deg}\left(\rho_{i}\right)=\omega\left(X_{\alpha^{(i)}}\right)$.

Proof. - When the set of indexes $I$ is finite, this version can be found in 4 . We show here that the result also holds when $I$ is infinite. First, observe that as $\left\{X_{\alpha^{(i)}}\right\}_{i \in I}$ is combinatorially free then $I$ is countable infinite.

For $i \in I$, put $Y_{i}=\varphi\left(\rho_{i}-1\right) \in \mathrm{E}_{1} ; n_{i}=\omega\left(Y_{i}\right)$. Let $\mathrm{I}(\mathrm{R}) \subset \mathrm{E}_{1}$ be the closed two-sided ideal of $\mathrm{E}_{1}$ topologically generated by the $Y_{i}$ 's, $i \in I$; one has $\operatorname{ker}(\theta) \simeq \mathrm{I}(\mathrm{R})$ (see for example [9. Chapter 7, §7.6, Theorem 7.17]). Let us recall now the topological G-isomorphism between $\mathrm{R} / \mathrm{R}^{p}[\mathrm{R}, \mathrm{R}]$ and $\mathrm{I}(\mathrm{R}) / \mathrm{I}(\mathrm{R}) \mathrm{E}_{1}$ (see for example [4, Proposition 4.3]). We want to some informations on the G-module $R / R^{p}[R, R]$, and then on $I(R) / I(R) E_{1}$.

For $i \in I$, let $Z_{i} \in \mathrm{A}$ be the initial form of $Y_{i} \in \mathrm{E}_{1}$ defined as follows: let us write $Y_{i}=Z_{i, n_{i}}+Z_{i, n_{i}+1}+\cdots$, where $n_{i}=\omega\left(Y_{i}\right)$ and where $Z_{i, j}$ are homogeneous polynomial of degree $j$ (eventually $Z_{i, j}=0$ ); then put $Z_{i}=Z_{i, n_{i}}$. Observe that $\widehat{\rho}_{i}=\widehat{Y}_{i}=\hat{Z}_{i}$.

Let $\mathrm{C}$ be the closed ideal of $\mathrm{A}=\mathbb{F}_{p}^{n c}\left[X_{1}, \cdots, X_{d}\right]$ generated by the family $\left\{Z_{i}\right\}_{i \in I}$. As the family $\left\{\hat{\rho}_{i}\right\}_{i \in I}$ is combinatorially free then by Theorem 1.3 the family $\left\{Z_{i}\right\}_{i \in I}$ is strongly free. Put $\mathrm{B}=\mathrm{A} / \mathrm{C}$.

Proposition 1.7. - One has $\mathrm{C}=\mathrm{Grad}(\mathrm{I}(\mathrm{R})) \subset \mathrm{A}$. In particular, as graded $\mathrm{A}$-modules, one gets $\operatorname{Grad}\left(\Lambda_{\mathrm{G}}\right) \simeq \mathrm{B}$, and

$$
\operatorname{Grad}\left(\mathrm{I}(\mathrm{R}) / \mathrm{I}(\mathrm{R}) \mathrm{E}_{1}\right) \simeq \mathrm{C} / \mathrm{CA}_{1} \simeq \bigoplus_{i \in I} \mathrm{~B} Z_{i} \simeq \bigoplus_{i \in I} \mathrm{~B}\left[n_{i}\right],
$$

where $\mathrm{B}\left[n_{i}\right]$ means $\mathrm{B}$ as $\mathrm{A}-$ module with an $n_{i}$-shift filtration.

Proof. - This is only a slightly generalization of the case $I$ finite; see proof of [4, Theorem 3.7].

Then by Theorem 1.3 and Proposition 1.7 we firstly get

$$
P_{\mathrm{G}}(t)=P_{\mathrm{B}}(t)=\left(1-d t+\sum_{i \in I} t^{n_{i}}\right)^{-1}
$$

Consider now the continuous morphism

$$
\Psi: \prod_{i \in I} \Lambda_{\mathrm{G}} \rightarrow \mathrm{I}(\mathrm{R}) / \mathrm{I}(\mathrm{R}) \mathrm{E}_{1} \simeq \mathrm{R} / \mathrm{R}^{p}[\mathrm{R}, \mathrm{R}],
$$


sendind $\left(a_{i}\right)$ to $\sum_{i} a_{i} Y_{i}\left(\bmod \mathrm{I}(\mathrm{R}) \mathrm{E}_{1}\right)$; as $n_{i} \rightarrow \infty$ with $i$, it is well-defined. Remember that $\Lambda_{\mathrm{G}} \simeq \mathrm{E} / \mathrm{I}(\mathrm{R})$. Put $\mathrm{N}=\operatorname{ker}(\Psi)$.

Lemma 1.8. - The map $\Psi$ is surjective.

Proof. - Put $\mathrm{W}=\left\{\sum_{i \in I} a_{i} Y_{i}, a_{i} \in \mathrm{E}\right\} \subset \mathrm{I}(\mathrm{R})$. Then

$$
\begin{aligned}
\mathrm{I}(\mathrm{R}) & =\mathrm{WE} \\
& =\mathrm{WF}_{p}+\mathrm{EWE}_{1}=\mathrm{W}+\mathrm{WE}_{1} .
\end{aligned}
$$

We conclude by observing that $\mathrm{WE}_{1} \subset \mathrm{I}(\mathrm{R}) \mathrm{E}_{1}$.

Therefore one gets a sequence of filtered G-modules:

$$
1 \rightarrow \mathrm{N} \rightarrow \prod_{i \in I} \Lambda_{\mathrm{G}}\left[n_{i}\right] \stackrel{\Psi}{\rightarrow} \mathrm{I}(\mathrm{R}) / \mathrm{I}(\mathrm{R}) \mathrm{E}_{1} \rightarrow 1
$$

This one induces the following sequence of graded A-modules:

$$
0 \rightarrow \operatorname{Grad}(\mathrm{N}) \rightarrow \operatorname{Grad}\left(\prod_{i \in I} \Lambda_{\mathrm{G}}\left[n_{i}\right]\right) \rightarrow \operatorname{Grad}\left(\mathrm{I}(\mathrm{R}) / \mathrm{I}(\mathrm{R}) \mathrm{E}_{1}\right) \rightarrow 0 .
$$

For the surjectivity, use the fact that $I$ is countable. Now as $n_{i} \rightarrow \infty$ with $i$, then

$$
\operatorname{Grad}\left(\prod_{i \in I} \Lambda_{\mathrm{G}}\left[n_{i}\right]\right)=\operatorname{Grad}\left(\bigoplus_{i \in I} \Lambda_{\mathrm{G}}\left[n_{i}\right]\right) \simeq \bigoplus_{i \in I} \mathrm{~B}\left[n_{i}\right] .
$$

By Proposition 1.7, we finally get that $\Psi$ induces an isomorphism between $\operatorname{Grad}\left(\prod_{i \in I} \Lambda_{\mathrm{G}}\left[n_{i}\right]\right)$ and $\operatorname{Grad}\left(\mathrm{I}(\mathrm{R}) / \mathrm{I}(\mathrm{R}) \mathrm{E}_{1}\right)$, which implies $\operatorname{Grad}(\mathrm{N})=0$, then $\mathrm{N}=0$. Hence, as G-modules, $\prod_{i \in I} \Lambda_{\mathrm{G}} \simeq \mathrm{I}(\mathrm{R}) / \mathrm{I}(\mathrm{R}) \mathrm{E}_{1} \simeq \mathrm{R} / \mathrm{R}^{p}[\mathrm{R}, \mathrm{R}]$, and we conclude with Theorem 1.1 .

Remark 1.9. - Conclusions of Theorem 1.6 also hold if $\left\{\hat{\rho}_{i}\right\}_{i \in I}$ is strongly free.

Remark 1.10. - For references on graded and filtered modules, see also [12, Chapter I and II].

1.1.4. Cup-products and cohomological dimension. - Here we suppose now $p>2$.

Let $\mathrm{G}$ be a pro- $p$ group of $p$-rank $d$ which is not pro- $p$ free. Recall that the cup product sends $H^{1}(\mathrm{G}) \otimes H^{1}(\mathrm{G})$ to $H^{2}(\mathrm{G})$. Labute in [10] gave a criterion involving cup-products so that $\mathrm{cd}(\mathrm{G})=2$. This point of view has been developped by Forré in [4]. Let us recall it.

Theorem 1.11 (Forré). - Let $p>2$ be a prime number. Let $\mathrm{G}$ be a finitely presented pro-p group which is not pro-p free. Suppose that $H^{1}(\mathrm{G})=U \oplus V$ such that $U \cup U=0$ and $U \cup V=H^{2}(\mathrm{G})$. Put $c=\operatorname{dim} V$. Then $\operatorname{cd}(\mathrm{G})=2$, and $\mathrm{G}$ can be described by some relations $\rho_{1}, \cdots, \rho_{r}$ such that the highest term of each $\rho_{i}$ can be written as $X_{t(i)} X_{s(i)}$ for some $s(i), t(i)$ such that $s(i) \leqslant c<t(i)$, and such that $(s(i), t(i)) \neq(s(j), t(j))$ for $i \neq j$.

Proof. - See the proof of [4, Theorem 6.4, Corollary 6.6] with the choice of the ordering $X_{1}<X_{2}<\cdots<X_{d}$.

Remark 1.12. - Observe that the family $\left\{X_{s(i)} X_{t(i)}\right\}_{i}$ of Theorem 1.11 is combinatorially free. 
Before to present a corollary, let us make the following observation: given $n \geqslant 1$, thanks to Example 1.5, one may find some $x \in \mathrm{F}$ such that the highest term of $x$ is like $X_{k} X_{j}^{n} X_{i}$ for $i<j<k$.

Corollary 1.13. - Consider the situation of Theorem 1.11. Suppose $c \geqslant 2$. For some fixed $1<i_{0} \leqslant c<j_{0} \leqslant d$, and $n \geqslant 1$, let $x_{n} \in \mathrm{F}$ of highest term $X_{j_{0}} X_{i_{0}}^{n} X_{1}$. Suppose moreover that $r<(d-c)(c-1)$. Then there exists $\left(i_{0}, j_{0}\right)$ such that the family $\left\{\widehat{\rho_{1}}, \cdots, \widehat{\rho_{r}}, \widehat{x_{n}}, n \geqslant 1\right\}$ is combinatorially free. In particular for such $\left(i_{0}, j_{0}\right)$ :

(i) the group quotient $\Gamma:=\mathrm{F} /\left\langle\rho, \cdots, \rho_{r}, x_{n}, n \in \mathbb{Z}_{>0}\right\rangle^{\text {Nor }}$ of $\mathrm{G}$ is of cohomological dimension 2;

(ii) $\operatorname{dim} H^{2}\left(\Gamma, \mathbb{F}_{p}\right)=\infty$;

(iii) The Poincaré series of $\Lambda_{\Gamma}$ is $\left(1-d t+r t^{2}+t^{3} \sum_{n \geqslant 0} t^{n}\right)^{-1}$.

Proof. - Thanks to Theorem 1.11, for $i=1, \cdots, r$, the highest term of $\rho_{i}$ is of the form $X_{t(i)} X_{s(i)}$ for some $s(i) \leqslant c<t(i)$, and the family $\mathscr{E}:=\left\{X_{t(1)} X_{s(1)}, \cdots, X_{t(r)} X_{s(r)}\right\}$ is combinatorially free. Now, as $r<(d-c)(c-1)$ and $c \geqslant 2$, we can find $\left(i_{0}, j_{0}\right)$ such that $X_{j_{0}} X_{i_{0}}$ is not in $\mathscr{E}$, and then $\mathscr{E} \cup\left\{X_{j_{0}} X_{i_{0}}^{n} X_{1}, n \in \mathbb{Z}_{>0}\right\}$ is combinatorially free. Then apply Theorem 1.6 .

Remark 1.14. - In fact $r \leqslant(d-c) c-2$ is sufficient. Indeed, with such condition one has $X_{j_{0}} X_{i_{0}} \notin \mathscr{E}$ for some $\left(i_{0}, j_{0}\right) \neq(1, r), i_{0} \leqslant c<j_{0} \leqslant r$. Hence, if $i_{0} \neq 1$ the family $\mathscr{E} \cup\left\{X_{j_{0}} X_{i_{0}}^{n} X_{1}, n \in \mathbb{Z}_{>0}\right\}$ is combinatorially free. Otherwise $j_{0} \neq r$, and take $\mathscr{E} \cup\left\{X_{r} X_{j_{0}}^{n} X_{i_{0}}, n \in \mathbb{Z}_{>0}\right\}$.

1.2. Arithmetic backgrounds. - Let $p$ be a prime number, and let $\mathrm{K}$ be a number field. For $p=2$, assume $\mathrm{K}$ totally imaginary. Let $S$ and $T$ two disjoint finite sets of prime ideals of the ring of integers $\mathscr{O}_{\mathrm{K}}$ of $\mathrm{K}$. We assume moreover that each $\mathfrak{p} \in S$ is such that $\left|\mathscr{O}_{\mathrm{K}} / \mathfrak{p}\right| \equiv 1(\bmod p)$; the set $S$ is called tame. We denote by $\mathrm{Cl}_{\mathrm{K}}^{T}(p)$ the $p$-Sylow of the $T$-class group of $\mathrm{K}$.

Let $\mathrm{K}_{S}^{T} / \mathrm{K}$ be the maximal pro- $p$ extension of $\mathrm{K}$ unramified outside $S$ and where each $\mathfrak{p} \in T$ splits completely in $\mathrm{K}_{S} / \mathrm{K}$; put $\mathrm{G}_{S}^{T}=\mathrm{Gal}\left(\mathrm{K}_{S}^{T} / \mathrm{K}\right)$. As we recalled it in Introduction, when $\mathrm{G}_{S}^{T}$ is infinite, the extension $\mathrm{K}_{S}^{T} / \mathrm{K}$ is asymptotically good. Recall Shafarevich's formula (see for example [5, Chapter I, §, Theorem 4.6]):

$$
d_{p} \mathrm{G}_{S}^{T}=|S|-\left(r_{1}+r_{2}\right)-1-|T|+\delta_{\mathrm{K}, p}+d_{p} \mathrm{~V}_{S}^{T} / \mathrm{K}^{\times p},
$$

where

$$
\mathrm{V}_{S}^{T}=\left\{x \in \mathrm{K}^{\times}, x \in \mathrm{K}_{\mathfrak{p}}^{p} U_{\mathfrak{p}} \forall x \notin S \cup T, x \in \mathrm{K}_{\mathfrak{p}}^{p} \forall \mathfrak{p} \in S\right\},
$$

and where $\delta_{\mathrm{K}, p}=1$ if $\mathrm{K}$ contains $\mu_{p}$ (the $p$-roots of 1 ), 0 otherwise. Here as usual, $\mathrm{K}_{\mathfrak{p}}$ is the completion of $\mathrm{K}$ at $\mathfrak{p}$, and $U_{\mathfrak{p}}$ is the group of the local units at $\mathfrak{p}$. Observe that if there is no $p$-extension of $\mathrm{K}\left(\mu_{p}\right)$ unramified outside $T$ and $p$ in which each prime of $S$ splits completely, then $\mathrm{V}_{S}^{T} / \mathrm{K}^{\times p}$ is trivial: this is a Chebotarev condition type.

Schmidt in [15] showed that $\mathrm{G}_{S}^{T}$ may be mild following the terminology of Labute [10]. More precisely, he proved:

Theorem 1.15 (Schmidt). - Let $\mathrm{K}$ be a number field and let $p$ be a prime number. For $p=2$ suppose $\mathrm{K}$ totally imaginary. Let $S_{0}$ and $T$ two disjoint finite sets of prime ideals of $\mathrm{K}$ with $S_{0}$ tame. Assume $T$ sufficiently large such that $\mathrm{Cl}_{\mathrm{K}}^{T}(p)$ is trivial; when $\mu_{\mathfrak{p}} \subset \mathrm{K}$, assume moreover that $T$ contains all prime ideals above $p$. Then there exist 
infinitely finite tame sets $S$ containing $S_{0}$ such that $H^{1}\left(\mathrm{G}_{S}^{T}\right)=U \oplus V$ where the two subspaces $U$ and $V$ satisfy: $(i) U \cup U=0 ;($ ii $) U \cup V=H^{2}\left(\mathrm{G}_{S}^{T}\right)$. Moreover, for such $S$ and $T$ one has $\operatorname{dim} H^{2}\left(\mathrm{G}_{S}^{T}\right)=\operatorname{dim} H^{1}\left(\mathrm{G}_{S}^{T}\right)+r_{1}+r_{2}+|T|-1$.

Theorem 1.15 is not presented in this form in [15], here we give the form we need: the result presented here can be found in the proof of Theorem 6.1 of [15].

At this level, let us compute the value of $c=\operatorname{dim} V$ of Theorem 1.15, following [15].

When $\mu_{p} \notin \mathrm{K}$ let us choose first a finite set $S_{0}$ of prime ideals of $\mathrm{K}$, tame and disjoint from $T$, such that for every $\mathfrak{p} \in S_{0}$, one has

$$
d_{p} \mathrm{G}_{S_{0} \backslash\{\mathfrak{p}\}}^{T}=\left|S_{0}\right|-r_{1}-r_{2}-|T|+\delta_{\mathrm{K}, p},
$$

which is equivalent by Shafarevich's formula to the triviality of $\mathrm{V}_{S_{0} \backslash\{\mathfrak{p}\}}^{T} / \mathrm{K}^{\times p}$.

When $\mu_{p} \subset \mathrm{K}$ let us choose $S_{0}$, finite, tame and disjoint from $T$, such that the set of the Frobenius at $\mathfrak{p}$ in $\mathrm{G}_{T}^{p-e l}$ when $\mathfrak{p}$ varies in $S_{0}$, corresponds to the nontrivial elements of $\mathrm{G}_{T}^{p-e l}$, where $\mathrm{G}_{T}^{p-e l}$ is the Galois group of the $p$-elementary abelian extension $\mathrm{K}_{T}^{p-e l} / \mathrm{K}$ of $\mathrm{K}_{T} / \mathrm{K}$. Here one has also the triviality of $\mathrm{V}_{S_{0} \backslash\{\mathfrak{p}\}}^{T} / \mathrm{K}^{\times p}$.

The set $S$ of Theorem 1.15 contains $S_{0}$, and is of size $2\left|S_{0}\right|$; the prime ideals $\mathfrak{p} \in S-S_{0}$ are choosen by respecting some global conditions, thanks to Chebotarev density theorem. Moreover $U=H^{1}\left(\mathrm{G}_{S_{0}}^{T}, \mathbb{F}_{p}\right)$, and the subspace $V$ is such that $\operatorname{dim} V=c=\left|S_{0}\right|$. See [15. Proof of Theorem 6.1] for more details.

Now observe the following:

Lemma 1.16. - Above the previous conditions, each prime $\mathfrak{p} \in S$ is ramified in the p-elementary abelian extension $\mathrm{K}_{S}^{T, p-e l} / \mathrm{K}$ of $\mathrm{K}_{S}^{T} / \mathrm{K}$.

Proof. - Observe first that if $S^{\prime \prime} \subset S^{\prime}$, then $\mathrm{V}_{S^{\prime}}^{T} / \mathrm{K}^{\times p} \hookrightarrow \mathrm{V}_{S^{\prime \prime}}^{T} / \mathrm{K}^{\times p}$.

Hence thanks to the choice of $S_{0}$, it is not difficult to see the following: for every $\mathfrak{p} \in S$, $\mathrm{V}_{S \backslash\{\mathfrak{p}\}}^{T} / \mathrm{K}^{\times p}$ is trivial. Then by Shafarevich's formula, we get that

$$
d_{p} \mathrm{G}_{S}^{T}=1+d_{p} \mathrm{G}_{S \backslash\{\mathfrak{p}\}}^{T},
$$

showing that $\mathfrak{p}$ is ramified in $\mathrm{K}_{S}^{T, p-e l} / \mathrm{K}$.

Put $\alpha_{\mathrm{K}, T}=3+2 \sqrt{2+r_{1}+r_{2}+|T|}$. In Theorem 1.15 one may take $S$ sufficiently large so that $d=\operatorname{dim} H^{1}\left(\mathrm{G}_{S}^{T}, \mathbb{F}_{p}\right)>\alpha_{\mathrm{K}, T}$.

Lemma 1.17. - If $d>\alpha_{\mathrm{K}, T}$, then $d+r_{1}+r_{2}+|T|-1<(d-c)(c-1)$ for every $c \in[2, d]$.

Proof. - Easy computation.

Let us finish this part with an obvious observation thanks to class field theory.

Remark 1.18. - If $\mathrm{G}_{S}^{T}$ is not trivial and of cohomological dimension at most 2, then $\operatorname{cd}\left(\mathrm{G}_{S}^{T}\right)=2$. 


\section{Example and proof}

2.1. Example. - - Take $p>2$, and $K=\mathbb{Q}$. In this case the relations of the pro- $p$ groups $\mathrm{G}_{S}$ are all local, and then not difficult to describe: this is the description due to Koch [9, Chapter 11, §11.4, Example 11.11].

Let $\ell$ be a prime number such that $p \mid \ell-1$. Denote by $\mathbb{Q}_{\ell}$ the (unique) cyclic degree $p$-extension of $\mathbb{Q}$ unramified outside $\ell$; the extension $\mathbb{Q}_{\ell} / \mathbb{Q}$ is totally ramified at $\ell$.

Let $S=\left\{\ell_{1}, \cdots, \ell_{d}\right\}$ be $d$ different prime numbers such that $p$ divides each $\ell_{i}-1$. The pro$p$ group $\mathrm{G}_{S}$ can be described by $x_{1}, \cdots, x_{d}$ generators, and $\rho_{1}, \cdots, \rho_{d}$ relations verifying:

$$
\rho_{i}=\prod_{j \neq i}\left[x_{i}, x_{j}\right]^{a_{j}(i)} \bmod \mathrm{F}_{3},
$$

where $a_{j}(i) \in \mathbb{Z} / p \mathbb{Z}$; moreover the element $x_{i}$ can be chosen such that it is a generator of the inertia group of $\ell_{i}$. The element $a_{j}(i)$ is zero if and only the prime $\ell_{i}$ splits in $\mathbb{Q}_{\ell_{j}} / \mathbb{Q}$, which is equivalent to

$$
\ell_{i}^{\left(\ell_{j}-1\right) / p} \equiv 1\left(\bmod \ell_{j}\right)
$$

- Typically take $p=3$, and $S_{0}=\{7,13\}, T=\varnothing$. Then put $S=\left\{p_{1}, p_{2}, p_{3}, p_{4}, p_{5}\right\}$ with $p_{1}=31, p_{2}=19, p_{3}=13, p_{4}=337, p_{5}=7$. Then the highest terms of the relations (1), viewed in $\mathbb{F}_{p}^{n c}\left[X_{1}, \cdots, X_{5}\right]$, are $\hat{\rho}_{1}=X_{1} X_{3}, \widehat{\rho_{2}}=X_{2} X_{4}, \widehat{\rho_{3}}=X_{2} X_{3}, \widehat{\rho_{4}}=X_{1} X_{4}, \hat{\rho_{5}}=$ $X_{1} X_{5}$. Hence as the $\hat{\rho}_{i}$ 's are combinatorially free, then $\mathrm{G}_{S}$ is of cohomological dimension 2 by Theorem 1.6 .

Now for each $n \in \mathbb{Z}_{>0}$, let us choose a prime number $p_{n}$ of $\mathbb{Z}$ such that the highest term in $\mathbb{F}_{p}^{n c} \llbracket X_{1}, \cdots, X_{5} \rrbracket$ of its Frobenius $\sigma_{n} \in \mathrm{G}_{S}$ is like $X_{5} X_{4}^{n} X_{1}$ (which is possible by Example 1.5 or Corollary 1.13, see next section). Then consider the maximal Galois subextension $\mathrm{L} / \mathbb{Q}$ of $\mathbb{Q}_{S} / \mathbb{Q}$ fixed by all the conjuguates of the $\tau_{n}$ 's (this is the "cutting towers" strategy of $[7])$. Put $G=\operatorname{Gal}(\mathrm{L} / \mathbb{Q})$. Then the pro-3 group $\mathrm{G}$ can be described by the generators $x_{1}, \cdots, x_{5}$, and the relations $\left\{\rho_{1}, \cdots, \rho_{5}, \tau_{n}, n \in \mathbb{Z}_{>0}\right\}$ (which is not a priori a minimal set). By construction all the $p_{n}$ split totally in $\mathrm{L} / \mathbb{Q}$. Observe now that

$$
\left\{\widehat{\rho_{1}}, \cdots, \widehat{\rho_{5}}, \widehat{\tau_{n}}, n \geqslant 1\right\}=\left\{X_{5} X_{1}, X_{5} X_{2}, X_{4} X_{3}, X_{4} X_{2}, X_{5} X_{3}, X_{5} X_{4}^{n} X_{1}, n \in \mathbb{Z}_{>0}\right\},
$$

which is combinatorially free. By Theorem 1.6 the pro-3-group $\mathrm{G}$ is of cohomological dimension $2, H^{2}(\mathrm{G})$ is infinite, and $\mathbb{F}_{3} \llbracket \mathrm{G} \rrbracket$ has $\left(1-5 t+5 t^{2}+t^{3}\left(1+t+t^{2}+\cdots\right)\right)^{-1}$ as Poincaré series.

2.2. Proof of the main result. - - Let $p>2$ be a prime number, and let $\mathrm{K}$ be a number field. Let $S_{0}$ and $T$ two finite disjoint sets of prime ideals of $\mathrm{K}$, where $S_{0}$ is tame. Take $T$ sufficiently large such that $\mathrm{Cl}_{\mathrm{K}}^{T}(p)$ is trivial. When $\mathrm{K}$ contains $\mu_{p}$, assume moreover that $T$ contains all $p$-adic prime ideals.

First take $S$ containing $S_{0}$ as in Theorem 1.15, and sufficiently large such that $d>\alpha_{\mathrm{K}, T}$. Put $\mathrm{G}=\mathrm{G}_{S}^{T}$. Here $r=\operatorname{dim} H^{2}(\mathrm{G})=d+r_{1}+r_{2}-1+|T|$.

Let us start with a minimal presentation of $\mathrm{G}$ :

$$
1 \longrightarrow \mathrm{R} \longrightarrow \mathrm{F} \stackrel{\varphi}{\longrightarrow} \mathrm{G} \longrightarrow 1 \text {. }
$$

By Theorem 1.15 and Theorem 1.11 the quotient $\mathrm{R} / \mathrm{R}^{p}[\mathrm{~F}, \mathrm{R}]$ may be generated as $\mathbb{F}_{p^{-}}$ vector spaces by some relations $\rho_{1}, \cdots, \rho_{r}$ such that the highest terms $\hat{\rho}_{k}$ are like $X_{i} X_{j}$ for some $i \leqslant c<j$, where $c=\operatorname{dim} V$. Observe that as $\mathrm{G}$ is FAb then $c \in[2, d-2]$. 
Given $n \geqslant 1$, then the quotient $\mathrm{G} / \mathrm{G}_{n+1}$ is finite. Put $\mathrm{K}_{(n+1)}=\left(\mathrm{K}_{S}^{T}\right)^{\mathrm{G}_{n+1}}$. Let $a_{n} \in \mathbb{Z}_{>0}$ be an increasing sequence. Let $x_{n} \in \mathrm{F}_{a_{n}} \backslash \mathrm{F}_{a_{n}+1}$. By Chebotarev density theorem there exists some prime ideal $\mathfrak{p}_{n} \subset \mathscr{O}_{\mathrm{K}}$ such that $\sigma_{\mathfrak{p}_{n}}$ is conjuguate to $x_{n}$ in $\operatorname{Gal}\left(\mathrm{K}_{\left(a_{n}+1\right)} / \mathrm{K}\right)$. Here $\sigma_{\mathfrak{p}_{n}} \in \mathrm{G}$ denotes the Frobenius of $\mathfrak{p}_{n}$ in $\mathrm{K}_{S}^{T} / \mathrm{K}$. Now take $z_{n} \in \mathrm{F}$ such that $\varphi\left(z_{n}\right)=\sigma_{\mathfrak{p}_{n}}$. Hence

$$
z_{n} \equiv \sigma_{\mathfrak{p}_{n}}\left(\bmod \mathrm{RF}_{a_{n}+1}\right) .
$$

In other words, there exists $y_{n} \in \mathrm{F}_{a_{n}+1}$ and $r_{n} \in \mathrm{R}$ such that $z_{n}=\sigma_{\mathfrak{p}_{n}} y_{n} r_{n}$.

Let $\Sigma=T \cup\left\{\mathfrak{p}_{1}, \mathfrak{p}_{2}, \cdots\right\}$, and consider $\mathrm{K}_{S}^{\Sigma}$ the maximal pro- $p$ extension of $\mathrm{K}$ unramified oustide $S$ and where each primes $\mathfrak{p}_{i}$ of $\Sigma$ splits completely. Put $G_{S}^{\Sigma}=\operatorname{Gal}\left(\mathrm{K}_{S}^{\Sigma} / \mathrm{K}\right)$. Then

$$
\mathrm{G}_{S}^{\Sigma} \simeq \mathrm{G} /\left\langle\sigma_{\mathfrak{p}_{n}}, n \in \mathbb{Z}_{>0}\right\rangle^{\text {Nor }} \text {. }
$$

Here $\left\langle\sigma_{\mathfrak{p}_{n}}, n \in \mathbb{Z}_{>0}\right\rangle^{\text {Nor }}$ is the normal closure of $\left\langle\sigma_{\mathfrak{p}_{n}}, n \in \mathbb{Z}_{>0}\right\rangle$ in $\mathrm{G}_{S}^{\Sigma}$. Hence $\mathrm{K}_{S}^{\Sigma}$ satisfies (i) and $($ ii $)$ of Theorem $\mathrm{A}$. But observe now that

$$
\mathrm{G} /\left\langle\sigma_{\mathfrak{p}_{n}}, n \in \mathbb{Z}_{>0}\right\rangle^{\text {Nor }} \simeq \mathrm{F} /\left\langle\rho_{1}, \cdots, \rho_{r}, z_{n}, n \in \mathbb{Z}_{>0}\right\rangle^{\text {Nor }}=\mathrm{F} /\left\langle\rho_{1}, \cdots, \rho_{r}, x_{n} y_{n}, n \in \mathbb{Z}_{>0}\right\rangle^{\text {Nor }},
$$

as $\sigma_{\mathfrak{p}_{n}}$ and $x_{n}$ are conjugate.

Since the highest term of each $x_{n} y_{n}$ in $\mathrm{E}=\mathbb{F}_{p}^{n c} \llbracket X_{1}, \cdots, X_{d} \rrbracket$ is the same as the highest term of $x_{n}$, it suffices to choose the $x_{n}$ 's as in Corollary 1.13 which is possible: indeed as $d>\alpha_{\mathrm{K}, T}$ then by Lemma $1.17 r<(c-1)(d-c)$, for every $c \in[1, d-1]$. Thanks to Corollary 1.13, one gets $(i i i),(i v)$, and (vi) of Theorem A.

$(v)$ : by Lemma 1.16 each prime ideal $\mathfrak{p} \in S$ is ramified in $\mathrm{K}_{S}^{T, p-e l} / \mathrm{K}$, showing that $\tau_{\mathfrak{p}} \in \mathrm{G}$ is not in $\mathrm{RF}^{p}[\mathrm{~F}, \mathrm{~F}]$, where $\tau_{\mathfrak{p}}$ is a generator of the inertia group at $\mathfrak{p}$ in $\mathrm{G}$. As the $p$-rank of $\mathrm{G}_{S}^{\Sigma}$ is the same as the $p$-rank of $\mathrm{G}$, each prime $\mathfrak{p} \in S$ is ramified in $\mathrm{K}_{S}^{\Sigma}$. But as $\mathrm{G}$ is without torsion (because $\operatorname{cd}(\mathrm{G})=2$ ), necessarly $\left\langle\tau_{\mathfrak{p}}\right\rangle \simeq \mathbb{Z}_{p}$, and the structure of local extensions forces $\left(\mathrm{K}_{S}^{\Sigma}\right)_{\mathfrak{p}} / \mathrm{K}_{\mathfrak{p}}$ to be maximal.

- Assume $p=2$, and $\mathrm{K}$ be totally imaginary. Then Theorem 1.15 holds, but Theorem 1.11 does not. As explained by Forré in [4, Proof Theorem 6.4], one has to take two orderings to show that the highest terms of the relations $\rho_{1}, \cdots, \rho_{r}$ are strongly free. Now in this context the strategy of the approximation of elements $x_{n}$ by some Frobenius as in Corollary 1.13 also applies. Then by following the proof of Theorem 6.4 in [4], and by choosing the $x_{n}$ 's as in the case $p \neq 2$, we observe that the initial forms of the new relations $\left\{\rho_{1}, \cdots, \rho_{r}, x_{n}, n \geqslant 1\right\}$ are still strongly free. We conclude by using Remark [1.9 of Theorem 1.6.

\section{References}

[1] D. J. Anick, Non-commutative graded algebras and their Hilbert series, J. of Algebra $78 \mathrm{n} 1$ (1982), 120-140.

[2] D. J. Anick, Inert sets and the Lie algebra associated to a group, J. Algebra 111 (1987), $154-165$.

[3] A. Brumer, Pseudocompact algebras, profinite groups and class formations, J. of Algebra 4 (1966), 442-470

[4] P. Forré, Strongly free sequences and pro-p-groups of cohomological dimension 2, J. reine u. angew. Math 658 (2011), 173-192. 
[5] G. Gras, Class Field Theory, From Theory to practice, corr. 2nd ed., Springer Monographs in Mathematics, Springer (2005), xiii +507 pages.

[6] F. Hajir, C. Maire, Tamely ramified towers and discriminant bounds for number fields, Compositio Math. 128 (2001), 35-53.

[7] F. Hajir, C. Maire, R. Ramakrishna, Cutting towers of number fields, 2019, arXiv:1901.04354.

[8] Y. Ihara, How many primes decompose completely in an infinite unramified Galois extension of a global field?, J. Math. Soc. Japon 35 (1983), no4, 693-709.

[9] H. Koch, Galois Theory of $p$-Extensions, Springer Monographs in Mathematics, SpringerVerlag, Berlin, 2002.

[10] J. Labute, Mild pro-p-groups and Galois groups of p-extensions of $\mathbb{Q}$, J. reine u. angew. Math. 596 (2006), 155-182.

[11] J. Labute, J. Mináč, Mild pro-2-groups and 2-extensions of $\mathbb{Q}$ with restricted ramification, J. Algebra 332 (2011), 136-158.

[12] M. Lazard, Groupes analytiques p-adiques, IHES Publ. Math. 26 (1965), 389-603.

[13] P. Lebacque, Quelques résultats effectifs concernant les invariants de Tsfasman-Vladut, Ann. Inst. Fourier 65 (2015), no. 1, 63-99.

[14] J. Neukirch, A. Schmidt and K. Wingberg, Cohomology of Number Fields, GMW 323, Springer-Verlag Berlin Heidelberg, 2000.

[15] A. Schmidt, Über Pro-p-Fundamentalgruppen markierter arithmetischer Kurven, J. reine u. angew. Math. 640 (2010), 203-235.

[16] M. Tsfasman and S. Vladut, Infinite global fields and the generalized Brauer-Siegel theorem. Dedicated to Yuri I. Manin on the occasion of his 65th birthday, Mosc. Math. J. 2 (2002), no 2, 329-402.

February 26, 2020

Oussama Hamza, Ecole Normale Supérieure de Lyon, Université de Lyon, 15 parvis René Descartes, 69342 Lyon Cedex 07, France • E-mail : oussama.hamza@ens-lyon.fr

Christian Maire, FEMTO-ST Institute, Université Bourgogne Franche-Comté, 15B Avenue des Montboucons, 25030 Besançon Cedex, France

- E-mail : christian.maire@univ-fcomte.fr 